\title{
New genetic insights into pancreatic cancer
}

Few effective therapies are available for pancreatic cancer, resulting in a 5-year survival rate of $<5 \%$. Findings published in Nature from the International Cancer Genome Consortium provide novel insights into the genetic abnormalities that characterize pancreatic cancer, from the largest cohort of patients studied to date.

This prospective, observational study included 142 patients with early (stage I and II) sporadic pancreatic ductal adenocarcinoma (PDAC), the cause of $>90 \%$ pancreatic cancer, from more than 20 institutions worldwide. "A combination of next-generation exome sequencing and single nucleotide polymorphism profiling of paired normal-tumour DNA samples was conducted in 99 patients," explain Sean Grimmond and Andrew Biankin, lead authors. "These methodologies enabled the genome-wide detection of point mutations, insertions and deletions and copy number variants to generate a full catalogue of somatic genomic events in an individual cancer."

The paired tumour analysis revealed substantial heterogeneity in pancreatic cancer with 2,016 non-silent mutations. 16 genes were substantially mutated, including those previously reported (such as KRAS) and also novel genes involved

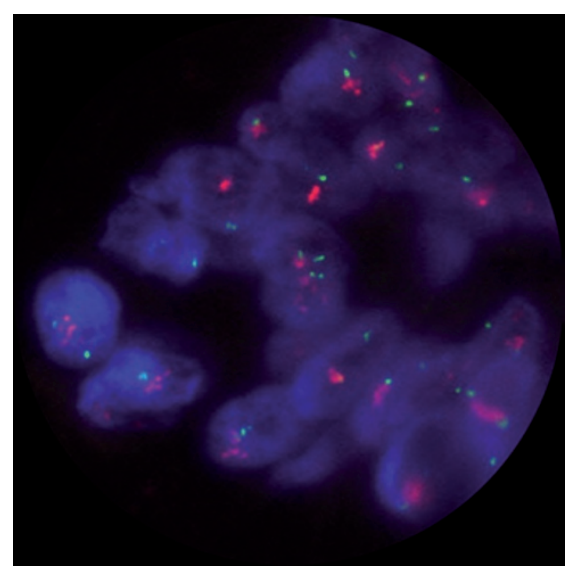

HER2 amplification in a patient with pancreatic ductal adenocarcinoma. Image courtesy of A. V. Biankin.

in chromatin modification (EPC1 and $A R I D 2)$, DNA damage repair (ATM) and other processes.

Functional screens of pancreatic cancer cell lines and data from mouse models provided further evidence for a role of these genetic mutations in carcinogenesis. Indeed, analysis of the core signalling pathways in PDAC (angiogenesis, apoptosis and cell cycle regulation) led to the identification of new mutated genes in these pathways. The researchers also discovered genetic aberrations in genes that encode regulators of axon guidance, a pathway important in CNS development. Further evidence from both in vitro studies and mouse models suggested that axon guidance genes have a role in pancreatic carcinogenesis.

New genetic tools have enabled characterization of cancers at the level of the individual. "We have generated xenografts and cell lines from the cancers that we sequenced. These models will facilitate the preclinical assessment of personalized therapeutic strategies to inform adaptive clinical trials," says Biankin. The Australian Pancreatic Cancer Genome Initiative has now launched an adaptive personalized medicine clinical trial in which patients with predefined actionable mutations are randomly assigned to undergo either standard therapy or a personalized approach. "These studies will, hopefully, enable refinement of current therapies as well as the development of novel therapeutic strategies for pancreatic cancer," they conclude.

\section{Katherine Smith}

Original article Biankin, A. V. et al. Pancreatic cancer genomes reveal aberrations in axon guidance pathway genes. Nature doi:10.1038/nature11547 